\title{
[ 416$]$
}

1Il. Veber einige Punkte in der Alomentheorie; yon Mrn. Dumas.

(Bescbluss.)

Iclı folireite zur Unterfuchung einiger Kiefol - und Bor-Verbindungen. Ich lıabe mich zunachlt mit dem Chlorkiefel") befchiftigt. Das, welches ich anwandto, war durch Einwirkung von Chlor auf ein Gemenge von Kiefelerde und Kohle, nach dem Verfaluren des $H_{r n}$. Oersted, orhalten. Um das überfohülfige Chlor zu entfernen, wurde es eine Zeit lang nit Queckfilber gelchuättelt, und darauf bei einer gelinden Warme deftillirt. In diefem Zuftande belals es die Durchficluigkeit, die Leichtflirfigkeit und das AufeJien doe Schivefelathers. Es fiedetc noch unter $100^{\circ} \mathrm{C}$. Folgendes find die Data und Refultate eines Verfinches.

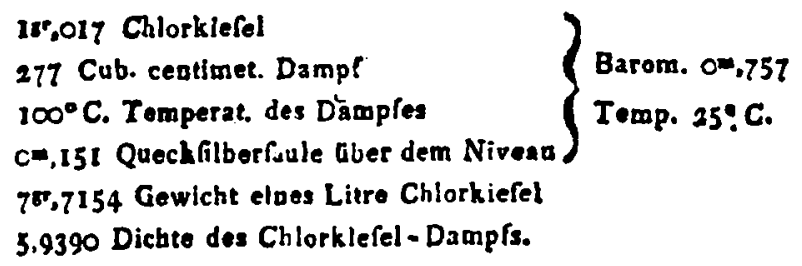

Nach den neutiten Verluchen des Hrn. Berzelins und in der Annalsme, dafs die Kiefelerde 3 Atone Saverftoff enthalte, liegt das Alomengewicht des Kiofels (Silicium) zwilchen 277,2 und 277,8. In diefer

-) Unter Táiefol if hier inmer, wle im Lehrbuche ron Berzolius, das Silicium zu vertehen. P. 


\section{[ 437 ]}

Hypothefe, wio leicht zu erlolien, wurde die. Dichto des Kiefeldampfes $=\overline{5}, 0597$ foyn und der Chlorkiefel aus einem Atome Kiefel und 6 Clulor beftehen. Nismlich:

$$
\begin{aligned}
& 6 \text { Vol. Chlor }=6 \times 2,470=14,820 \\
& 1 \cdot \text { Kiefel }=\cdots \cdot \cdot \frac{3.0 \% 97}{17.8797}
\end{aligned}
$$

Aber $\frac{17,9797}{3}=5,9599$, woraus offcubar folgen würde, dafs 6 Vol. Chlor und a Vol. Kielel fich bei der Yerbindung auf 3 Vol. verdichten.

Man gelangt zu einfacheren Verliălnjiten, wenn man dag Atomengewiclit des Kiefels auf das Drittel reducirt, wodurch es $=92,5$ wird. Alsdann hat man. 1,0ıg7 für die Dichle des Kieleldampfes, $\mathrm{Si}+\mathrm{O}$ lïr die Zulanmenletzung der Kielelerde, und $\mathrm{Si}_{+}+\mathrm{Cl}_{1}{ }^{3}$ für dio deg Chlorkielels. In dieler Hypothele hat man

$$
\begin{aligned}
& 3 \text { Vol. Chlor }=2,47 \times 2=4,97 \\
& 1 \text { Vol. Kiefel }=. \cdot \cdot=1,0197 \\
& \hline \text { Vol. Chlorklefel . . . }=5,9597
\end{aligned}
$$

Da ich iudefs hoffe, über kurz oder lang za gonauen Begriffen über diefe Art von Verlıältuiten zu gelangen, fo will ich lieber diel'o Fragen unentfchie. den laffen, als Aanderungen unmotivirt vorfclslagen. Ich nelime indel's die letztero diefer Zahlen an, weil fie die folgendon Rechnungen einfacher machen. Wir wollen überdiefs verfuchen, wie diefe Refultate mit denen fitimmeu, die fich aus der Zufammenfetzung und Dichte dor Hilufs - Kielelfuure ableiten IaTen. Was die Zuliumnonfelzung derlelben betriff, fo ift fio durch die Verfuche des Hrn. Berzeliua befímint, und 


\section{[ 4.8 ]}

wird durch das folgende Refillat beftatigt. Wenn man trocknes Hufekiefallaures Gas über erlitzien Baryl gehen lalst, fo fundet oine lebliafte Abforbtion Slatt, hegleitet von einer Erglühung, dio fo ftark iff, dals die Malfo fich verglaft. Das Droduct ift graulich weifs. Es entweicht kein Gas bei dem Verluche. Selor reiner Baryt wurde vor und nacl der Abforbtion gewogen, und dalurch Folgendes gefunden:

$$
\begin{aligned}
& \text { beobacht. Refultat berechnet. Refultat } \\
& \text { Baryt • . 85,62 . - 85,44 } \\
& \text { Flufskiefelfure } 14.38 \text {. . I } 14.56
\end{aligned}
$$

Das bereclınete Relultat fiützt fich auf die Annahme, dafs ein Atom Baryt fich nuit 2 Atome Kiefel und - Alonien Fluor verbinde. Die Uebereinftimmnng zwilchen dem beobachleten und berechneten Reliultate, und die Gewifsheit meinerfeits, dals in diefem Verlizche das Gas keine theilweife Zerletzung erlitt, fondern olune Rückfiand ablorbirt wurdo, beweifen, dals die Flufskiefeltäure wirklich a Atom Kielel und 2 Atone Flnor enthălt, was übrigens felır gut mit den Verfuclıen des Hrn. Berzelius übereinftimmt. Hieraus orgiebt fich für die Dichtı diefes Gafes:

$$
\begin{aligned}
& 1 \text { Vol. Kiefel }= 1,0197 \\
& 2 \text { Vol. Fluot }= 2.5776 \\
& \hline 3.5973
\end{aligned}
$$

Hr. John Davy hat 3,5735 gefunden, ein Refultat, das von dein meinjgen wenig abwoiclit. Ifier die Data und Relultate meiner eignen Beobuchtungel! über diefen Gugenffind :

285,635 Gewicht des leoren Ballons

288.733 Gewicht des Ballons ron trocknar Luft

296,690 - - - - Flufskiefelfaure 


\section{[ 419 ]}

woraue dis Dichte dieles Galfes $=3,600$. Hr. John Davy wirirdo ohne Zwoifol ein gennueres Reliultat erlralton hatien, wesn er feinen Varfuch mit einem betrichtlicheron Volunien vom Gafe angeftellt hatte.

Ich laabe oben die Zufanmenfelzung diefoo Körpers in der Annahme berechuset, dafo er aus Fluor und Kiefel gebildet fey. Hr. Berzelius betrachtet ilın als eine Verbindung von Flul'slaure und Kiefolerde. Obgleich ich mir vorgenoninien habe, fpaterhin anf die Gefchichte des Fluors zurückzukehren, fo will ich hier doch die Zufammenfetzung feiner Hauptverbindungen nacle der von mir angenonmenen Botrachtungsw eife anfïliren.

Hr. H. Dary hat gefunden, dafs 100 Thl. Fluorcalcium i75 Thl. trocknen lchiwefelfuuren Kalk geben. Bei Wiederholung dieles Verfincices liatte Hr. B e r zofi us anfangs andere Refiultate erhalten; faterhin hat aber diefer berülumte Chemilier feine erfen Angaben berichtigt und diefelbon Zallen, wio Hr. Davy, erhalten \%. Nach diefen findet man:

$$
\begin{aligned}
& \text { Calcium - 52,27 oder } 1 \text { At. }=512,06 \\
& \text { Fluor - } 47,73 \cdots \cdot 4 \text { At. }=467,53 \\
& \text { Fluorcalcium } 100 \cdots \cdot \text { At. }=979,04
\end{aligned}
$$

woraus folgt, dafs das Atomengevicht deo Fluors 726,89 und dio Diolitigkeit deffelben 2,288 ift.

7) Man darf jedoch daraus nieht fehliofson, dafs Hrn. Da vy's Analyfo dio riehtige war. Hr. D. uberfah die Phosphorfiure und das Manganoxyd in dem zur Analyfe angewantien FlufsSpathe voll Derbyshire, Es wur alfo nur Znfall, defa diefer Fehler von den tbrigen fatt compenfizt wurde.

P. 


\section{[ 420 ]}

Bei diofem Gegenftando hat man nur zu wallen zwifchen der Zulammenfetzung, welcho anl die Analogio nut dem Chlor gegründet ift, und der, wolcho man erhalt, wonn nau die Fluorverbindungen auf Seito der Schwefelvorbindungen ftellt. Der Hanptverfuch von Hrn. Davy Jalst fich auf glejche Art naclı beiden Hypothelien erkluren. Derfelbe lah, dafs flnorwaferftoflaures Ammoniak, mit Kalium behandolt, 1 Vol. Wafferfroft und 2 Vol. Ammoniak lieferte. Man erhatt ein alinliches Relultat, man mag das ang gleichen Volumen Ammoniak und Clulorwalferftofliuure gebildete Ammonialifalz zerfetzen, oder das, welches aus der Veroinigung von 2 Vol. Anmoniak und a Vol. Scli wefel watterftoffliture entlpringt, d.h. das gewöhnliche fclıwetel wa ferfioflfaure Ammoniak.

Ich laabe das aus der Analogie nuit den Clilore abgeleiteto, alo das walır chlieinlichere, angenonmen. Wern unan diele Hypolluefo anf die vou Hrn. Berzelius zerlegten Verbiudungen onwondet; findet nam dielelbon viel einfacher und leichter zu ftudiren.

Zunâchlf hat man für die Zulaumenfetzung der Flulgkielielfäure:

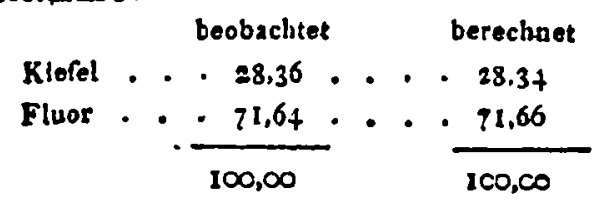

Bei dem bereclineten Refultate find dio obigen Atomengowichte des Fluors und des Kiefels angenommen : day beobachtete hefultat ift aus deu Verfuchen des $\mathrm{Hrn}$. Berzelius abgeleitet.

Nimmt man ein Alom Sanerftoff in der Kiefolfäure an, lo werden die weiterhin aufzuftellenden Re- 


\section{[ 421$]$}

Sullate viel einfacher. Wollte man ubbrigens eine andere Hypothefo annehnen, fo wäre es leiclit, diefelben umzuwandeln.

Bringt man dio Flufskiefelfaure mit Wafler zufanmen, fo erleidet be eine theilweile Zerletzung, welche von den Hru. J. Davy und Hrn. Berzelins unterfucht worden if. Ich will mich niclit in das Einzelne der Verfuche einlallen, ich will blols zeigen, dafs es bei der vorliegenden Betrachtungsart leichter ift, die Refultate 24 überlehen \%. Man fieht in der That, dafs, nach Hrn. Berzelins Verfuchen, ein Drittel des in Gafe euthaltenen Siliciums in Kiefelerde übergeht und fich ausfcheidet. Nimmt nıau alfo 3 Atome Fluorkiefel, fo folgt, dafs zwei Atome unzerfetzt bleiben und dal's ein At. durch Wirkung des Walfers Fluorwafterftoffliure und Kiefelerde giebt. Die Kiefelfaure lagert Gich ab und die andern Stoffe vereinigen fich zu einem Guorwaferftoffauren Fluorkiefel, in welchem jedes Atom Flnorwalferftoffluro verbuncin ift mit einem Atom Fluorkiefel. Die Formel für diefe Verbindungen ift allo: $\mathrm{FH}+\mathrm{SiF}^{2}$.

Man überfielıt die Erfcheinungen dieler Reaction beffer in der nachftelienden Formel:

$$
3 \mathrm{SiF}^{2}+\mathrm{H}^{2}=\mathrm{Si}+\left(2 \mathrm{FH}+{ }_{2} \mathrm{SiF}^{2}\right)
$$

Die fogenannten Doppelfalze von flufsfaurer KieCelerde, d. h. diejenigen Salze, welche man or-

") Es if wahrfcheinlich Hrn. Dumas nicht bekanne, dafs He. Berzelius felbft, im erften Theile reines Lehrbuchs (Dresden 1825), alle vol ihm bei der Flufsfrure beobachteten Thatfachen, der Theorie der Wafferfoffiuren gemals, alfo gerado to wio hior, befchriebet and erklurt bat.

P. 


\section{$\left[\begin{array}{ll}422\end{array}\right]$}

lialt, wenn man fluorwaflerfofflauren Fluorkiefel mit Bafen behandelt, find nun leiclit en clafificiren. Es ift einleuchtend, dafs die Fluorwallerftontfrure und die Bafe ein Flnormetall und Waller geben. Diels Fluormetall verbindet fich uit dem Fluorkiefel und fpielt gegen denfelben die Rolle einer Bafe. Diefe Doppel-Fluorure haben die gröfste Aehnlichkeit mit den Doppel - Cyanuren.

Die Reaction des fluorwafferftofflauren Fluorkiofels auf die Bafen lafst fich nach der folgenden Formel bereclinen:

$$
4\left(\mathrm{FH}+\mathrm{SiF}^{2}\right)+\ddot{\mathrm{R}}=\left(\mathrm{RF}^{4}+\mathrm{Si}^{2}\right)+2 \dot{\mathrm{H}}^{2}
$$

d. h. es entftehen daraus Doppel -Fluorïre, in denen der Fluorkiefel nothwendig zwei Mal lo viel Fluor enthalt als das andere Fluorüre. Diefe Anzeige ift für alle Rechnungen hinlanglich. Man nehme z. B. das flufsfaure Kiefelerde-Natron des Hrn. Berzelius. Bereclunet man feine Analyle als Fluorkiefel und Fluornatrium, fo findet man:

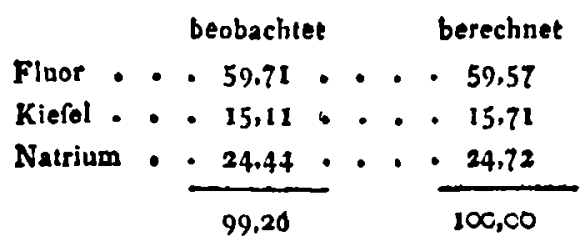

Aohnliche Refultate erhalt man beim flufsfauren Kicfelerde-Baryt, den HIr. Berzelius gleichfalls anaJyfirt liat.

Ich will diefe Erörterung nicht weiter fortfetzen; fie reicht hin, um die Hauptreactionen des Fluors zu bezeichnen; aber fie zeigt zugleich, dal's, oblchon diefer Körper fich in vicler Hinfioht dem Chlor und Jod 


\section{$\left[\begin{array}{lll}4 & 423 & ]\end{array}\right]$}

z.u nuhern fcheint, es andere Beziehnngen giebt, durch dio er fich auf Seite deg Schwefels fiellt, welcher letztnre eine grolse Menge von Doppel-Verbindungen giebt.

Mein Hauplzweck ift, die bekannten Verbindungen des Bors mit denen des Kiefels zu vergleichen.

Das Bor ift unter den einfachen Körpern derjenige, welcher, in bezug auf die Verhaltnifle bei feinen Verbindungen, die merkwürdigften Eigenthümlichkeiten zeigt. Da die Cheniiker diefen Stoff nur in fehr geringen Mengen rein erhalten konnten, fo haben uns die direclen Verfuche auch nur wenig genaue Angaben geliefert. Dio Unterfuchung einiger Verbindungen der Borfaure hat zwar Hrn. Berzelius neuerlich zu felır genauen Refultaten gefülhrt; ich hoffe indefs, dafs die Vorfuche, welche jch logleich vorlegen werde, die wahren Verlualtnille diefes Körpers und leiner Hauptverbindungen auf eine weniget zweideutige Weile feftftellen werden.

Durch die Verbrennung deg Bors hat fich $\mathrm{Hr}$. Berzelius überzengt, dafs dieler Körper, $11 \mathrm{~m}$ in Borfaure überzugehen, ungefuhr das Doppelte feines Gewichts an Sauerftoff anfnimmt. Die Saure muls alfo in Hundert wenigftens 66 Sauerftoff enthalten. Die, nach dema treffliclien Verfalıreu des Hrn. A rfvedso n gemachte, Aualyle des Boraxes gab ihm folgendo Refultale :

$$
\begin{aligned}
& \text { Borfäure - } 36,59 \\
& \text { Natron . } 16,31=4,1715 \text { Sauerftoff } \\
& \text { Waffer } \cdot \frac{\cdot}{4,10}=41,889 \\
& \text { Borax . - } 100
\end{aligned}
$$




\section{[ 434 ]}

Der Sanerftoff des Walfers betragt offenbar das Zehnfaclio vou denu des Natrours. Will man die kleine Differenz in der Beobachtung corrigiren, lo ift klar, dafs diefe, niberdiel's felir kleine, Correction an Nalron vollzogen werden mufs, defen Monge durch fie kaum geandert wird, walırend man eine betrăclitlichere Differenz haben wïrde, wein man fio beim WalTer anbrüchte. Diefemnach hat man für die Zufammenfotzung des Boraxes :

$$
\begin{aligned}
& \text { Borfaure . 30,5247 • } 100 \\
& \text { Natroa • 16,3753 • 44,8336 } \\
& \text { Water - 47,1000 } \\
& \text { Borax • . } 100 .
\end{aligned}
$$

Da 44,83̄̄6 Natron an Sauerftoff 1s,4684 enthalten, fo muiften 100 Thl. Saure, welche durch diefo Menge Bafis gefattigt wird, 68,8 1 Sauerftoff enthalten, d. l. das Sechsfache; denn dieles Mulliplum nahert fich am meiften den durch directe Oxydation erhaltenen Refultaten.

Die Borfuuro kann fich überdiefs in fehr verfchiedenen Verlaltnifen mit den Bafen verbinden, wovon Hr. Berzelius nacls leinen und des Hrn. Arfvedson Analyfen melirere Beil'piele enfuilırt. 100 Thl. Surure fätigen in den verfchiedenen Salzen Quantitaten von Bafen, die enthalten:

5.73+ Samerfoff. Doppelt borfaures Kali und Natron 11,468 - - Borax und neutrales borfaures Ammoniak I 7,202 - - Boracit und anderthalb bafifch borfaures Ammoniak

22,93 - - Doppolt bafifch borfaures Kall (darch Schmelsung)

34,40 - - Dreifach bafifch borfaures Natron (durch Schmalsung) und Ammosiak. 


\section{$\left[\begin{array}{lll}425 & 1\end{array}\right.$}

Da.die Ssure 68,8, Sauerftoff enthalt, fo fielit man; dafs diele Menge das 12-, 6-, 4-, 3-oder 2-facho des Sanerftoffgelialts der Bafen in den verfohiedenen Salzen ift; und da die gewöhnlich ften Verbindungen diejenigen find, in welchen der Sauerftoff der Săuro das 6 - oder 12 -fache ift, fo lohlielst Hr. Berzeliue darans, dafo die Saure 6 Atome Sanerftoff und 2 Atom Bor enthallt.

Unter diefem Geficlitspunkte würde die Boraxfäure analog feyn der Cluromlaure, der einzigen von den bekann!eu Süuren, in der man 6 Atome Sauerftoff und 1 Atom Hadical varanggefetzt hat. Es ift indefo wahrCcheinlich, dal's diefe Zulammenfetzung weder für dio eine, noch für die andere dieler Săuren dio richlige iff. In der That., wir wollen einige Borverbindungen unterfuchen: wir werden bald lehen, dafs die angenommene Hypothefe unhaltbar ift.

Als ich trocknes Clilor übcr ein glühnendes Go. menge von Kollle und Borfuture gehen liefo, erhielt ich einen gafigen Körper; den ich bald als das dem Fluobor (der Flufsborfaure) entfprechendo Chlorbor erkannte. Icls wufgte nicht, dafo diefes Gas fchon bekannt war. Hr. Thenard, dem jch meine Beobachtung miltheilte, forderte mich auf, fie dem Inftitute vorzulegen. Es wurdo in der Sitzung am 15. Mai ${ }_{1826}$ eine Notiz uber dielen Gegenftand vorgelefen nnd darauf in die Annales de Chimie et de Physin que eingerûckt. Einige. Tage hernach erfulir ich, dafs $\mathrm{H}_{r}$ Berzelius in feiner. Fchönen Arbeit über dio Flulsfaure fich mit dem. Bor befcläfligt habe: Aus Unachtfamkeit, olme Zweifel, ift derjenigo Thoil feines Ablandlung, weloher fich auf diefen Körper boAnnal. d. Phyfik. B. 85, St. 3. J. 182 \%, St, 3.

E. 


\section{? 4261}

zieht, in der franzöfilchen Ueberfetzung ausgelaflen; aber er findet fich in den englifchen Journalen ( $\int_{0}$ wio in d. Annal. Bd. 78. S. 123. 1 .), und aus diefon habo ich die obigen Naclırichten gefclsöpft. Im Laufe feiner Verfuche hat Hr. Berzeliug das Bor der Wirkung des trocknen Chlors ausgeletzt, und auf diele VWeile eine gafige Verbindung erlialten, die durchaus mit der von mir dargeftellten identifch ift. Ilim verdankt man allo diefo Entdeckung. Ich gehe jetzt zur Unterfuchung der Eigenfcliaften diefer Verbindung über.

Iclı fuchte zunächlt das Verhältnils aufzufinden zwifchen dem erzeugten Chlorbor und dem Kohlenoxyd, das durch die Verbindung des Sauerftoffs der Borfaure mit der Kohle des Gemenges entftanden war. Ich fuchte hierauf die Diclite des Chlorbors zu beftimmen. Durch oinen felur fonderbaren 'Zufall fand ich bei meinen erften Verfuchen nahe gleiche Volumina von Kolılenoxydgas und von einem in Wafter lüsliclien Gafe. Ich hielt das letztere fiir reines Chlorbor. Zugleich fand ich durch ein Mittel aus zwei Verfuchen die Dichte des löslichen $\mathrm{Grafes}_{\mathrm{a}}=\mathbf{5}$,42. Das Sonderbare in diefem Refultate war, dafo es für die Borfüure eine $Z_{\text {nl- }}$ fammenfotzung gab, identifch mit der, welcho die HH. Gay-Lussac und Thenard in ihren "Recherches physico-chimiques" fanden, nämlich 33 Saucrfioff in Hundert, wahrend Hr. Barzelius 68 Sauerfioff in Hundert annimmt. Bei Wiederlsolung und $\mathrm{Ab}$ anderang diefer Verfuche fand ich bald oine Fohlerquelle, dio jch überfehen hatte. Diefe liogt darin, dafo Gch eine ziemlich grofse Menge von Clulorwalterftoffluturo mittellt des Walfers oder Wafterfioffs des Korkftöpfels bildet, welchø gleichzeitig fo- 


\section{[ 427$]$}

wohl die Menge des löslichen Gafos rermehrt; als ancly feine Dishte verm indert. In einem, mit anfserfier Sorgfalt angeftellten Verlitclie, bei welchem ich den Strom des Chlors felir langfam gehen liefs und das Porcellanrolir eine Stunde lang ganz verfchloten erhitzt hatte, bevor ich den Chlor-Apparat anlegte, konnte ich einen Augenblick abpalen, worin fich dio Gafe in ihren wahren Verhälnifen entwickelten. Die Analyle gab Folgendes:

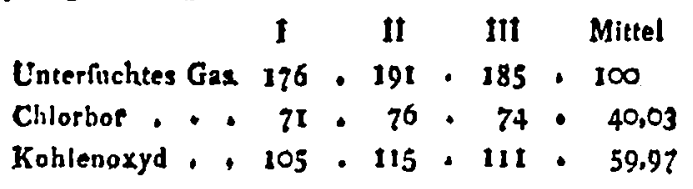

Ehe ich diefe 3 Probegläfer voll Gas auffing, hatte ich die Luft vollkommen ausgetrieben; allein es war unmöglich, die Gaserzengung länger fortzufezzen, weil die Korkftöplel anfingen Wafterlioff abzugeben, was man an der fortfchreitenden Vermelırung des inı Waffer löslichen Gales erkannte. Das Verhaltnifs $2: 3$, welches in den obigen Analylen fichtbar ift, wurde alsdann geftört und die verhaltnifsmafsigen Mengen des löslichen Gafes und des Kohlenoxydgafes wurden ganz unregelmalsig. Ich würde kein Vortranen auf diefes Rofultat legen, hatte mir nicht der Verfuch bei melirmaliger Viederholung ahnliche Re. foltate gegeben. Ich betrachte es alfo als gewifs, dafo das Chlor bei Einwirkung auf ein Gemenge von Borfure und Kohle, 2 Vol, Chlorbor und 3 Vol. Kohlenoxyd giebt. - Uebrigens wird die Bildung der Clslorwailerftofffurure beftandig dadurch angezeigt, dafs fich langs dem Wege des Gafes oine ziemlich betrachtlich. Menge Borfäuro ablagert. 


\section{[ $\begin{array}{lll}428 & \text { ] }\end{array}$}

Der Apparat, welchen iclı bei diefen Verfuchen anwende, befteht ans einer Porcellanröhre, welche das Gemenge enthalt, . und bis zum Rolliglühen erhitzt wird. Das eine ihrec Enden nimmt das trockne Chlor anf, an dem andern befindet fich ain:Varftolo, und daran wioder oin gebogenes Rolir, welcheo unter Qneck filber mündet. Ich erhitze eines Zeit Jang das, mit leinem Pfropfen verfehene Porcellanrohr, um die Feuchtigkeit, welche im Gemenge oder im Apparate zurückgeblicben feyn könnte, zul vertreiben. Ich laffe jetzt das trockne.Clalor eintreten, und wenn der Gasfirom recht im Gange ift und eine Viertelfitunde lang angehaltien liat, lege ich den Vorftols und das gekrïmmte Rohr - vor. Der Vorftofs bekleidet ficlt bald mit einem weifsen $\mathrm{Ab}$ falze, welcher, in Geftalt von leichten Flocken, auch in das Rolur übergeht und daffelbe zaweilen verftopft. Man mufs alfo Rölıren bereit lıalten, um fie nötligenfalls zn wechfeln. Bei Unterfuchung diefes Ablatzes fand fich derfelbe zufammengefelzt ans viel Borlüure, ans ein wonig Chloralumium und zuweilen ans einer melir oder weniger betrăchtlichen Menge Chloreifen, welche beide Stoffe olnne $Z$ weifel von einer Unreinlieit der Kolıle oder der Sauro herrührten. Da nun eine wefentliche Eigenfehaft des Chlorbors darin befteht, dafs es bei Einwirkung von Wafter in Borfuture und Salzfare umgewandelt wird, , fa ift klar, dafo die Gegenwart der Borfauro in dem Abgelagerten eine verhaltnifsmafoigo Menge Chlorwafterftoffiunro in dem Gafo anzeigt. Als ich dio oben angeführten Meffungen machte, bildete ficl keine Wolke, weder in dem Yorftofs noch in der Rōhre; aber es war unmög- 


\section{[ 429 ]}

liah; die.Operation: lange fortzufetzen, ohne dafs nicht diofe. Reaction meltr oder weniger ftark aufirat.

Nachdem jeh alle diefe Umftinde wohl aufgehrellt lialle, nalm ich die Dichte des Chlorbors wieder vor, und ich fand 3,3̆ fär die Dichte des im Wafter lösliclien Theils des gewogenen Gemenges. Als ich aber die durch die Bedingungen deo Verfucho angezeiglen Correctionen vollzog, anderte fich diefer Werth und wurde viel grölsar. Wie ich beim Verluche und bei der Rechnung verfulir, zeigt Folgendes.

I:b wandro einen Bailon an, deffen Rauminhalt war $=21,174$

Voll trochner Luft bei $24^{\circ} \mathrm{C}$. und $0 \mathrm{~m}, 758 \mathrm{wog}$ er . 283,750

Leer, vor Elnführung des Gales • • • . 281,250

Voll des Gasgemenges . . . . . . 256,050

$\mathrm{Da} 2^{1}, 174$ trockiner Luft unter den angeführten Umfiinden 28r,584 wiegen, fo findet man, dafs obr,084 Luft vor Einfiphrung des $G$ afes in dem Ballon befindliclt waren, und dals das Gemenge 4rr,38+ wog. Iclı öfnete nan den Ballon unter Wafter. Alle darin lojlichen Galo verfchwanden, und'es blieb ein Rückftand von 1',097, befteliend aus Luft und Kolnlenoxyd. Folgendes wasen die Volumina der einzelnen Gafe, reducirt auf $0^{\circ}$ C. und $0^{\infty}, 76$, und die entfprechenden Gowichte:

01,065 atmofpharifthe Luft.. $=08 r, 084$
1,032 Kohlenoxyd .... . $=1,304$
0,306 Chlorwaffertoffraure. . $=0,495$
0,586 Chlorbor ..... $=\frac{3,001}{48,884}$

Diefs gefetzt, fo findet man $5^{\mathrm{gr}, 1212}$ alo Gowioht eines Litres Chlorbor und 3,9+2 als Dichta dellelben begen die Luft. Die Mengo der Chlorwafferftofflaure 


\section{[ 430 ]}

labe ich nach folgender Betrachtung berechnet. Abgefelien von der. Lnft, mufste der Ballon 1,934 rosu Gasgemenge euthalten. Darin fand ich. $1^{1}, 0 j: 2$ Kohlenoxyd; diefs giebt $0_{1}, 8_{92}$ lösliches. Gas. Aber diefer Menge Kohlenoxyd ent Pprechen nur ol,683 Chlorbor; es hatte fich allo Chlorwafterftofflaure bei der Bereitung dos Gafes erzengt. Um die Menge dorfelben zu berechnen; nahm ich an, dafs ein Volumen Chlorbor hălte 3. Vol. Chlorwalferfioffiuure liefern müßfen. Diưs zeigten dio hijer erörterten Beobachtungen. Deun, wenn durch Einwirkung der Kohle und des Chlors auf die Borfaure 2 Vol. Chlorbor und 3 Vol. Rolulenoxyd entftehen, und andrerfeits bei der Zerfetzung des Chlorbors durch Waffer Clslorwafterftoffluture und Borfäıre, fo muls man notlıwendig dio folgenden Verhültuife auffellen :

$$
\begin{aligned}
& 3 \text { Vol. Kohlonoxyd }=\left\{\begin{array}{l}
1,5 \text { Vol. Sauerfoff } \\
1,5 \text { Vol. Kohlondampf }
\end{array}\right. \\
& 2 \text { Val. Chlorbor }=\left\{\begin{array}{l}
3 \text { Vol. Chlor } \\
1 \text { Vol, Bor }
\end{array}\right.
\end{aligned}
$$

Und wenn Chlorbor auf Vifer wirkt, wird es geben; 2 Vol. Chlorbar $=\left\{\begin{array}{l}3 \text { Vol. Chlor }+3 \text { Vol. Wa Werferfoff }= \\ 6 \text { Vol. Chlorwafferfoefthure } \\ 1 \text { Vol. Bor }+1,5 \text { Vol. Sauerfof }=\text { Borfauro. }\end{array}\right.$

Diefe Verhaltuife find die einzigen, die fich aufftellen lafen; denn das in 2 Vol. Chlorbor enthaltene Bor hat die anderthalb Yol. Sanerftoff liefern gemurgt, die fich im Kolslenoxyde befinden, und kann fie, bei Einwirkung auf Walfer, nur durch Mitwirkung von 3 Vol. Chlor wiedor aufnehmen. Diolo Verlualtuito find alfo nothwondig, und wir werden fehen, dafs diq Erfalirung fio auf eine entfclioidende Woile beftatigt. 


\section{[ 431 ]}

Hr. Borzelius hat gefunden, dafs die Boraxfaure in Hundert 68,8, Sacuerftoff enthalt. Nimmt man mit mir an, dal's diefe Săure aus 2 Vol. Bor und 3 Vol. Sauerftoff gebildet ift, fo hat man in der That 31,19 : $68,8_{1}:: 2 x: 300$ und daraus $x=67,91$ als Atomengowicht des Bors. Nach diefem Gewichto findet man 0,7487 für die Dichte des Bordampfes. Für das Chlorbor werden wir nach diefen Angaben haben:

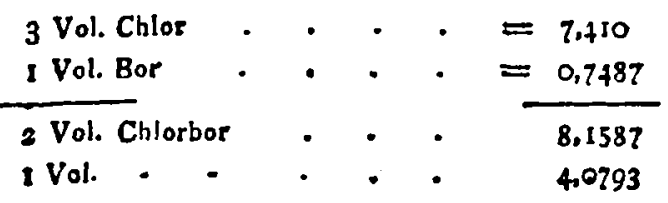

Die bereclinete Dichte des Chlorbors würde alfo 4,07g3 feyn. Diefs weicht wenig ab von 3,942, was die Erfahrung gab, vor allern, wonn man die Feluler in Betraclit zieht, dio unvermeidlich mit dem von mir angewandien Verfaliren verbunden find. Der Unterlchied würde walırlchicinlich grölser gewefen leyu, hatte ich nicht dic ängftliche Sorgfalt auf den Verluch gewandt und dadurch, trotz der zu überwindenden Schwicrigkeiten, ein zuverlaffiges Refultat erlialten.

Ich habe verfchiedene Methoden zur Bereitung des Chlorbors erdacht und verficlit; aber keine ift mir ge. glückt. Ich erhitzte gepulverte Borfiure mit concentrirter Schwefelfiure und warf Stïcke gefchmolzenen Kochlalzes in das Gemenge. Es entwickelte fich nur Clilorwaiterftomliuregas. - Ich erlitzte ein Gemengo von Kochfalz und zuvor gefclimolzener Borlaure in einer Porzellanretorte bis zur Weilsgluth. Es entwickelten fich uur einige Blafeu Chlorwafterftoffliure. 


\section{( 4332 I}

Nachdem das Feuer melhrere Stundenilang untorlialten worden war, liefs ich dio Ketorto erkalten. Ichs fand in derlelben dic Saure und das Salz in zwoi Schichten felır deutlich von einander gefondert. Hasto eine Heaction Statt finden kömnen, fo würde diefer Umftand fie niclıt gehindert haben, donn fie würdo in den Berührungspunkten beider Schichlen eingetreten feyn. - Ich erhitzlo in einer Glasretorte, bis zur vollen Sclımelzung, ein Gemenge von 4 Thl. gofclimolzonem lauren fchwefelfauren Kali, 2 Thl. gofclimolzonem Kochfalze und 1 Thl. gefclimolzener Borfäure. Ich erhiolt nur Chlor und lchlwellige Sünre, beladen mit Dainpfen von walferfreier Schwefelfaure. Ich glaube nicht, dals fich Chlorbor gebildet hatte. In allen Fallen aber kominte diefs Verfaliren von keinem Nutzen leyn.

Die Refultate, welche das Chlorbor liefert, worden durch die, welcho man bei Unterfuchung der Fluoborfaure erhalt, vollkommen beftatigt. HinfichtJich der Dichto defelben habo ich Folgendes beobachtet :

$$
\begin{aligned}
& \text { 302,681 Gewicht des leeren Ballons } \\
& \text { 306,608 - - mit trockner Luft gefollien } \\
& \$ 11,762 \text { - - mit FluoborSiure gefüllted. }
\end{aligned}
$$

Hieraus findet man die Dichte des Gales $=2,3129$. Hr. John Davy fand 2,3709 , aber or berückfichtigte nicht die Fluorkiefriliure, welche in dem von ihm gewägton Gafe zugegen war. Das, welches ich anwandle, liefs bei Behandlung mit Waffer nur Borfaure fallen.

Erwagt man andrerfeils, ćafs Borfaure und Flutorwafterftofliaure fich duroh ilure gegenfeitigo Binwir- 


\section{[ 433 ]}

kung in Walter and Fluoborfauro unwandela, fo muls man haben:

$$
\begin{aligned}
& \left.\begin{array}{l}
\text { 2. Vol. Bor } \\
\text { 1.5- Sauerfof } \\
\text { erzeugen }
\end{array}\right\} \begin{array}{c}
\text { 1 Vol. Bor } \\
3 \text { Vol. Fluor } \\
-
\end{array} \\
& \text { s Vol. Fluor } \\
& \text { s Vol. WanterRoff } \\
& \left\{\begin{array}{l}
1 \text { Vol. Bor } \\
3 \text { Vol. Fluor } \\
\text { erzeugen } \\
3 \text { Vol. Waflerdampe. }
\end{array}\right.
\end{aligned}
$$

Die Fluoborfüure muls diefennach zufammengefetzt leyn ans 5 Vol. Fluor und 2 Vol. Bor. Aber

3 Vol. Fluor $=3.366_{4}$

$$
1 \text { Vol. Bor }=\frac{0,7+87}{4,6151} \text { and } \frac{4,6151}{2}=2,3075
$$

was mit dem beobachteten Refultate übereinftimmt. Das Fluoborliure - Cas enthylt alfo ein halbes Volumen Bor und anderthalb Volumina Fluor.

Vergleiclit man die Reliuliale, welche ich hier in Bezng auf den Kielel und auf das Lor aufgefülırt habe, fo fich! man, dali, wenn die Analogie, welche man zwifchen diefen Körpern und dem Kohlenftoffo auffellen gewollt, gegründet leyn foll, die von ilmen gebildeten Verbindungen andern Verbindungen der Kolle ent Prechen mü గen.

Die Kieliclerde l'cheint ein oder zwei Atome Sauerftoff zu enthalten und nuliert lich dadurch der Kol lenliure.

Die Borfuure l'cheint alus 1 oder a Vol. Bor und 3 Vol. Sanerftoff gebildet, und nuhert foch in diefem Falle der Oxalfăure.

Diefe Analogien lafen fich mit Sicherheit nur durch eine antinerkfame Vergleichung der kiefelfauren, kolulonlauren, borfauren und oxalliauren Salze 


\section{[ 434 ]}

feffftellen. Ich wage keino beftimmte Meinung übor dielen Gegenliand zu fallen, überzeugt, wie ich fchon bemerkt habe, dafo une für vielo diefer Falle die nöthigen Angaben follen, un derartigo Zulammenftellungen zu begründen.

Ich will diefe Abhandlung mit einem Vergleiclıo des Chlorzinns und Chlortitans befchliefsen. Ich finde in diefen beiden Verbindungen eine gleicho Volumenanzalıl auf glejche Wieile verdiclitet. Durch diefo Aehnliclıkeit wird die Bezichung befiatigt, welclie $\mathrm{Hr}$. Mitscherlich unter denlelben nachgewielen bat.

Zinn. Der Spiritns Libavii greift immer das Queckfilber an, aber nicht vermöge eines et waigen Golinlles von überfchüfrigem Chlor, fondern dadurch, dal's er in einfaches Chlorzinn ïbergeht und Chlorqueckfilber im Min. erzeugt. Da diele Reaction, welcho allgeinein 212 fegn fcheint, fich dennoch auf felır kleine Mengen befchränkt, fo liabe ich den Verluch mittelft des Apparats yon Hrn. Gay - Lussac gemacht. Bei Wiederlsolung deftelben habe ich faft identilcliso Refultate erhalten, und bei denen, welche ich hier anfïhre, war der Angriff auf das Queckfilber nur durch ein fehr dünnes Häutchen nerklich, welches deffen Oberflacho bedeckte. Ich lege das vollfte Vertrauen in die erlaltenen Relultate, obgleich wegen dieler goringen Veranderung die Dichte ein wenig zu grofs gefunden wurde.

Der Spiritus Libavii fiedet, unter einem Drucke von $0 ", 767$, bei $120^{\circ} \mathrm{C}$, die Data und Refultato des Verliuclis waren folgende: 


\section{[ 435 ]}

25r,352 Spirtus Libavil

322 Cubikcentimetr. Dampf (om,759 Barom.

$\left.\begin{array}{l}\text { 00,079 Qneckfilberfiule } \\ \text { I24 C. Temperatur des Dampis }\end{array}\right\} 26^{\circ}$ C. Temp.

II 12.9514 Gewlcht eines Litres

9,1997 Dichto des Dampfer.

Mit dem von Hrn. Berzeli us gegebenen Atomengewichte des Zinns, 3470,58 , findet man 16,215 fïr die Dichto des Zinndampfo. Wir mälen alfo haben:

$$
\begin{aligned}
& \text { IVol. Zinndampf . = }=16,215 \\
& 8 \text { Vol. Clilor. . . = } 19,760 \\
& \hline 35,975
\end{aligned}
$$

Nan ift $\frac{35.975}{4}=8,993$, was dem erhaltenen Refallafe fohr nalie kommt, allein eine wenig walırfcheinliche Art der Verbindung vorausletzt, die|nünulich, dal's 8 Vol. Chlor und a Vol. Zinndampf an 4 Vol. verdichtet feyen. Reducirt unan das Atomengewicht des Zinns auf die Halfte, fo bloibt die Sclwwierigkeit faft die nämliche, denn man würdo laben 4 Vol. Chlor und, Vol. Zinndampf verdiclitet zu 2 Vol. Ich glaube allo, dars nıan das von Hrn. Berzelins gegebeno Atomengewicht auf das Viertel reduciren mufs. Eg wird alsdann $366_{i}, 6_{4}^{\prime}$ und die Dichte des Zinndampfo $=4,053$. In diefer Hypothefe hat man für das Doppelt-Clulorzinn

$$
\begin{aligned}
& \text { IVol. Zinndampf } \cdot \dot{ }=4,053 \\
& 2 \text { Vol. Chlos } \cdot \dot{*}=4,940 \\
& \hline \text { Vol. Doppeli-Chlorzina }=8,993
\end{aligned}
$$

Diefemnach würdo das Zinnoxyd a Atom Zinn und Atom Sauerftoff, $S t+O$, euthalten und 


\section{$\left[\begin{array}{lll}436 & ]\end{array}\right.$}

das Zinnoxydul a Atome Zinn und a Atom Sauerfoff; $8 t^{2}+0$.

Titan. Dio Analogio zwifclien dem Titan und Zinn, welcho von Hru. Mitsclierlich nach des Ifomorplie ihrer in der Natur vorkommenden Oxyde aufgeftellt, und von Hrn. H. Rose in feinen Verfuchen über das Titan entwickelt worden ift, hat nicls zu einigen Verluchen veranlal'st, mir Chlortitan zu verlchliaffell. Ich wandte anfangs reines Titanoxyd an und erhielt damit auch völlig reines Clulorlitaii. Das Oxyd, genifclit mit einern Viertel leines Gewiclts an wolilgetrockneter Kolule, wurde bis zur Rothgluth in eineml'orzellanrolurc erlitzt und durcl diefes ein Strom von trocknem Chlor geleitet. In dem Ballon und dern Vorftofse, welche mit der Röhre verbunden waren, fah man vom erften Augenblicke des Verfuchs an eine farblofe Flüffigkeit fich verdichten, die an der Luft aufserordontlich rauchte und felır fluclitig war. Diefe war das Clalortitan. Die Eigenfchaften dellelben zeigten bald, dafs es fich leicht rom Chlorcifen im Maximo trennen lafe, und dafs man es folglich leicht und in Menge aus dem natürlichen Titanoxyd bereiten köune. In der That, als diefs Oxyd gepülvert mit einesu Viertel oder einem Drittel leines Gewichis an Kohlo genifcht und wie das reine $O_{x ;}$ d behandelt wurde, erhielt man in der Verlangerung und im Ballon viel Chloreilen, welches fich den Wänden des Gefafses anhing, und viel flülighes Clilortitan, das man durch Abgiefsen falt rein ablondern konnte. Es entJielt dennoch ein wenig Chloreilen, aber keinesweges gelöft, londern als Flitterchen, die lich bei Ruho abletzen. 


\section{(1) 432]}

- Sonrerhalten; iff das Chlortitan niemalg rollkomment faxblos, foin .Geruch, dentet auf.Gegenwart von Chlor und feine gelbliche Farbe befturkt diefen Verdacht. Eudlich, wenn man es in Wafter löft, enthalt dieles, aufser dem gewöhnlichen clilorwalferfiofffauren Titan, Chlor gelöft, welches. Lackmuspapier bleiclit.

Man reinigt es leicht, indem snan es mit kleinen Mengen von Queckfalber fchüttelt und darauf 2 bis $\mathbf{j}$ Mal in einer kleinen Retorte über ein.weng diefes Metalles abzielıt. Man erlıalt es alsdann völlig fáılos und durchlichtig. Es wirkt dann niclit nehr auf Queckfilber, und bildet mit Wafler chlorwallerfiofffaures Titan olıne überfahülifiges Chlor.

Hr. E.S.George liat (Ann. of Philos. Jan. 1825 . p. 18. dief. Ann. Bd. ig. S. 171) ein Chlortitan kenuen gelelırt, das durch unmittelbare Ein wirkung des Clılors anf das erhitzte Metall bereitet war. Diefs Chlortitan if dem eben von mir befchriebenen in melirerer Bezielıung ahnlich, würde jedoch nach Hrn. G. ein Perchlorure feyn, das durch Einwirkung des Waffers ein gewöhnliches chlorwallerfiofflaurc's Salz lieferto und dabei die Hilffe feines Clikors verlöre. Ich wage nicht, über diefen Gegenftand zu entfclieiden. So lange das meinige überichüfhiges Chlor entlielt, war es gelb, befafs den Geruch von Chlor und liefs bei Wirkung des Wafers viel von diefenr Stoffe ent weichen. Durch mehrmalige Defijllation, felbh ohne Queckfilber, nahm fein Chlorgeruch und feine gelbe Farbe betrâchtlich ab.

Das farblofo Chlortitan fiedet, unter dem Drucke vou om, 763 , bei $135^{\circ}$ C. . Seine Dichto. ift gröfser alo 


\section{[ 438 ]}

dio dee Waltors. Dis. Data und Refultate eines. Werfnches linfichtlich der Dichto. leines Dampfos'waronfolgende:

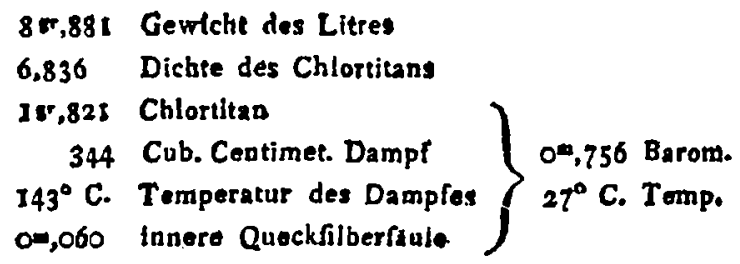

Hr. H. Rose hat das Atornengewicht des Titans hauptfäclilich dadurch beftimmt, da er er das Schwofeltitan durch Verbrenunng in der Lnft in Oxyd verwandelte. Er Ichlielist ans leinen Verfuclien, dals diefo Gewicht 778,2 Sey. Es reducirt fich anf 764,6 , wenn man in der Rechnung 200 fïr das Atomengewicht des Scliwefels nimmt, Itatt 201,16. Diefs letztere Gewiclit giebt 8,430 für die Dichle des Titandampfes. Aber diele Beftimmung fetzt 4 Atome Sauerftoft im Oxydo voraus; und diefen würden $8 \mathrm{At}$. Clulor in Clulortitan entfprechen. Hieraus folgt:

$$
\begin{aligned}
& 1 \text { Vol. Titandampl . . }=8.430 \\
& \$ \text { Vol Chlor . . . }=19.760 \\
& 28,190
\end{aligned}
$$

Aber $\frac{28.190}{4}=7,047$, ein Refultat, das fich foli? dem vorhergehenden nahert. Man hatte alfo, wie für das Zinn, g Vol. zu 4 Vol. verdichtet. Aus gleichon Gründen, wio für jenes Metall, reducire ich das Atomengewicht auf das Viertel. Es wird dadurch 191,15. Dio Dichte des Titandampfo wird 2,107, und die Titanfaure oder das weifos Tilenoxyd enthalt alodann 


\section{[ 439]}

1. At. Metall und at. Sanerftoff. Was das Clilortitan betrift, fo ift es gebildet aus:

$$
\begin{aligned}
& \text { IVol. Titandampf . . }=2.307 \\
& 2 \text { Vol. Chlor . . = } 4,940 \\
& \text { I Vol. Chlortitan . . = }=7,047
\end{aligned}
$$

Es ift durchaus nütligg, neue Verfuche. über das Atomengewicht des. Titans zu maclien, un zwilchen den Refultaten des Hrn. Rose und den meinigen zu onticheiden *). Die Analyfe des Chlorlitans bietet in diefer Hinficht ein felir genames Mittel dar. Ich habe nicht Zeit gehabt, mich mit diefer zu befchiuftigen.

Icl kann aus den in diefer Ablandlung enthaltenen Thatfachen nicht eher fichere Schliuffe ziehen, als bis ich die Diclite des Scliwefels, Plıosphors, Arlenihs, Selens und isaliums direct beflimnit habe. Icl h hin mit dicfen Verfuchen befchuftigt und werde nachfiens dio Relultate bekannt maclien. Sie befeftigen vielleicht den Gefichtspunkt, unter welchem man das Gefetz von Hrn. Gay - Lus sac über die Ammoniakverbindungen und das von den HH. Dulong und Petit überdie fpecifilchen Wărmen zu hetrachten liat.

Ich werde in der Folge das Refultat meiner Vurfuche über eine gewifle Zalıl von Clilormetallen bekannt machen, unter welchen ich mich heute begnü-

-) Durch dle Analyre des Chlortitans lafst fich offenbar das Atomengewicht des Tilans weit genarer beftimmed, als durch die Verbrennung des Schwefeltitane 24 Titanfsure. Aus diefrm Grunde hat auch Prof. H. Rose fehon vor ainiger Zelt die Analyfe des Chlortitans unternommen, and wird diefelbe nachone bekanat machen. 


\section{[ 440 ]}

ge, nar ein fehr flichtiges Clilormangan anzufthren, das der Manganfaure ent [pricht. Ich hofie duroh Vern einigung aller dieler Angaben, weun auch nicht meinen $Z$ weck zu creiclien, doch wenigftens mich demfelben fo weit zu nahern, als es der Luftand der WifSchaft erlaubt.

Ehe ich diefe Abliandlung fehliefse, mafs ich bemerken; dafs Hr. Duliong ficl, l'chon feit langer Zeit vorgenommen hat, dic Diclite des Schwefeldampfes direct zu beftimmen. Er liatte die Güte gehabt, nich mit dem zn diefem Verluche befimmiten $A$ pparate bekannt zu machen. Er weielit ganzlich ab von dem, welchen ich vorher belohirieb. Denuoch würde jch den Schwefel niclit auf die Lifte derjenigen Körper gebracht haben, nit dones ich die Akademie zu unterhalten gedenke, lälle or mir nicht unerwarteto Befonderheiten gezeigt, welche vielleicht den Verfuch des Hrn. Dulong unausfülirbar machen.

Iclı füge hier eine Tafel übèr dic von mir direct beobacliteten Dicliten Jrinzu, und vereinige damit die aus ihnen abgeleiten Dichten einiger einfachen Körper. Icls bemerke indefs wiederliolt, dafs ich keinen grolsen Werth auf diofe Inductionen lege. Wir find von dem Zeitpunkle, wo die Molecularchemie fich nach fichern Regeln richten könnte, noch fehr weit entfernt, ungeàchtet der fehr grofsen Vortheile, welche diefer Theil der Naturkundo aus den Arbeiten der Hll. Cay-Lusaac, Borzolius, Dulong und Petit, Mitscherlich, fo wio aus den theoretifchen Betrachtungen der $\mathrm{HH}$. Ampere und Arogadro gozogen liat. Dio ungemeine Thatigkeit des Hru. Berzeliue und der gute 


\section{[44:]}

Geift der. Chemiker, mit denen er Deutfchland boreichert hat, laften indel's hinficlitlich diefes wiclitigen Gegenftandes eine baldige und dauerhafte Umwälzung erwarten,

Tafel ober die Dichte dez in dlefer Abhandlung unterfuchten Dăınpfe and Gafe, und über das Gewicht eines Litres derfelben bei $0^{\circ} \mathrm{C}$. und $0^{m}, 7^{6}$.

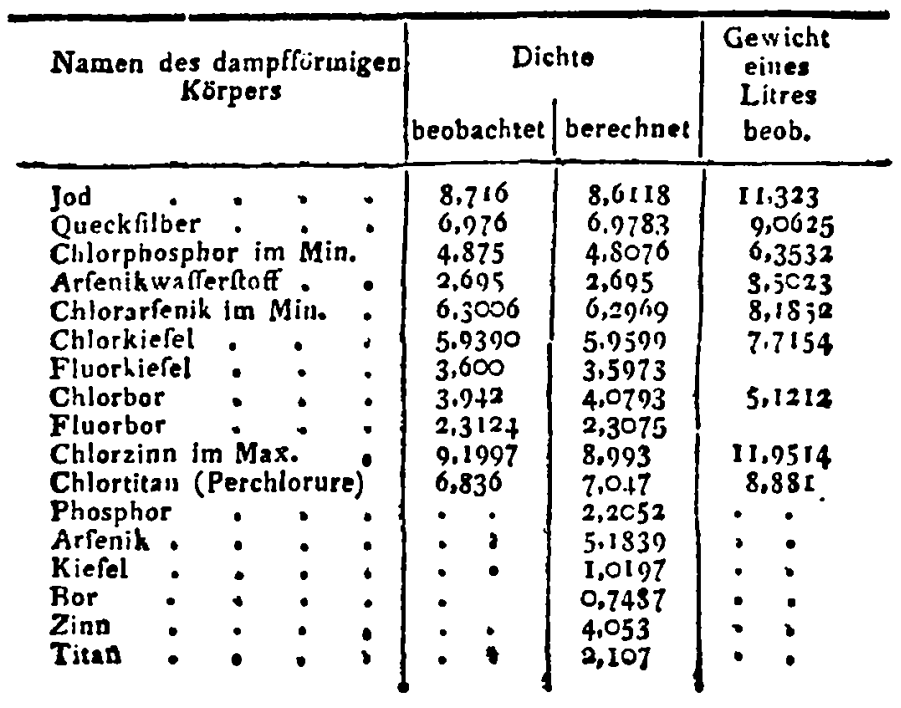

Berichtigung. Nach dem Sntze S. 293 (des vorlgen Heftes) Z. $₹$ v. u.: Dle Atomengewichte der elementaren K४rper fallen hierdurch Qberall mit den Dichten oder fpecifichen Gewichten ihrer Gafe oder Dampfe zufammen, - if hinzuzufetzen: - robald bet letzteren das fpeciffehe Gewicht des Sauerftofgafec zur binhoit angenommen wird. P. 